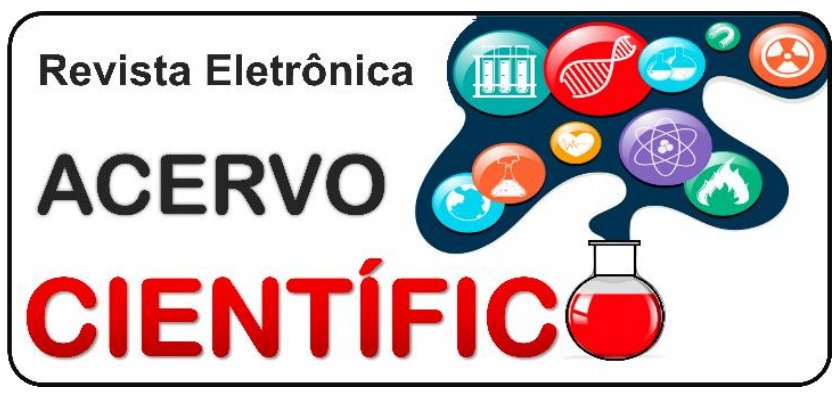

REVISÃO BIBLIOGRÁFICA

Recebido em: 6/2020

Aceito em: 7/2020

Publicado em: 11/2020

\title{
A importância do planejamento familiar e da anticoncepção no puerpério: uma revisão integrativa
}

\author{
The importance of family planning and anticonception in the puerperium: a integrative \\ review
}
La importancia de la planificación familiar y la anticoncepción en puerperio: una revisión integradora

Catharine Vieira Barbosa Eloy ${ }^{1 *}$, Marina de Pádua Nogueira Menezes ${ }^{1}$, Rafael Rezende Rocha de Oliveira ${ }^{1}$, Fabienny Bezerra Ribeiro' ${ }^{1}$, Juliane de Carvalho Rocha Moura'.

Resumo: Avaliar a importância do planejamento familiar no contexto do puerpério e ressaltar como a baixa adesão às práticas de planejamento familiar tornam-se prejudiciais ao binômio maternofetal, ocasionando intervalos intergestacionais pouco espaçados e gestações não planejadas. Trata-se de uma revisão integrativa acerca do impacto que o planejamento familiar e a anticoncepção no pós parto desempenham na qualidade de vida de puérperas e casais. O presente estudo baseou-se nos princípios metodológicos para busca de artigos nas bases de dados eletrônicas: MEDLINE e PubMed, com corte temporal de cinco anos (2015-2019). Para o rastreamento dos artigos indexados nas bases de dados foram utilizados os descritores: "planejamento familiar", "anticoncepção" e "puerpério". Incluíram-se 16 artigos, todos em inglês. Os artigos abordaram a importância do planejamento familiar e quais os fatores determinantes para diminuir as necessidades não atendidas de aconselhamento pós parto. O planejamento familiar (PF) pós parto constitui um importante instrumento nas práticas de saúde reprodutiva das mulheres. Apesar do déficit que existe quanto ao aconselhamento pós parto, o PF, quando bem orientado, fornece assistência de qualidade permitindo que, a mulher ou o casal, tenham autonomia para decidir sobre as próximas gestações.

Palavras-chave: Planejamento familiar, Anticoncepção, Puerpério.

Abstract: To evaluate the importance of family planning in the context of the puerperium and to emphasize how low adherence to family planning practices becomes harmful to the maternal-fetal binomial, causing narrowly spaced intergestational intervals and unplanned pregnancies. This is a integrative review of the impact that family planning and contraception in the postpartum period have to the quality of life of puerperal women and couples. This study was based on the methodological principles for searching for articles in the electronic databases: MEDLINE and PubMed, with a five-year time cut (2015-2019). The following descriptors were used to track the articles indexed in the databases: "family planning", "contraception" and "puerperium". 16 articles were included, all in English. The articles addressed the importance of family planning and what are the determining factors for reducing unmet postpartum counseling needs. Postpartum family planning (FP) is an important tool in women's reproductive health practices. Despite the deficit that exists regarding postpartum counseling, the FP, when well oriented, provides quality assistance allowing the woman or the couple to have autonomy to decide on the next pregnancies.

Keyword: Family planning, Contraception, Puerperium..

${ }^{1}$ Universidade Tiradentes (UNIT), Aracaju-Sergipe. *E-mail: barbosacath08@gmail.com

REAC/EJSC | Vol. 14 | e4274 | DOI: https://doi.org/10.25248/reac.e4274.2020 Página 1 de 10 
Resumen: Evaluar la importancia de la planificación familiar en el contexto del puerperio y enfatizar cómo la baja adherencia a las prácticas de planificación familiar se vuelve dañina para el binomio materno-fetal, causando intervalos intergestacionales estrechamente espaciados y embarazos no planificados. Esta es una revisión integradora del impacto que la planificación familiar y la anticoncepción en el período posparto tienen en la calidad de vida de las mujeres y parejas puerperales. El presente estudio se basó en los principios metodológicos para buscar artículos en las bases de datos electrónicas: MEDLINE y PubMed, con un corte de tiempo de cinco años (2015-2019). Se utilizaron los siguientes descriptores para rastrear los artículos indexados en las bases de datos: "planificación familiar", "anticoncepción" y "puerperio". se incluyeron 16 artículos, todos en inglés. Los artículos abordaron la importancia de la planificación familiar y cuáles son los factores determinantes para reducir las necesidades de asesoramiento posparto no satisfechas. La planificación familiar posparto (PF) es una herramienta importante en las prácticas de salud reproductiva de las mujeres. A pesar del déficit que existe con respecto a la consejería posparto, la PF, cuando está bien orientada, brinda asistencia de calidad que permite a la mujer o la pareja tener autonomía para decidir sobre los próximos embarazos.

Palabras clave: Planificación familiar, Anticoncepción, Puerperio.

\section{INTRODUÇÃO}

O puerpério é compreendido como o retorno do organismo materno ao estado pré-gravídico, tendo início desde a dequitação placentária até 6 semanas completas após o parto (ZUGAIB M, 2016). É um momento oportuno para abordar questões acerca do planejamento familiar e da anticoncepção, visto que, as mulheres tendem a visitar serviços de saúde mais regularmente neste período (SILEO KM, et al., 2015)

A busca por um atendimento integral durante o ciclo gravídico-puerperal é muito importante, apesar dessa assistência não ser de amplo acesso às mulheres. Mecanismos de referência e contrarreferência são falhos ou mesmo inexistentes, dificultando que medidas de planejamento familiar sejam adotadas. Abordar questões acerca da anticoncepção no pós-parto traz benefícios que melhoram a qualidade de vida dessas mulheres e reduz de forma significativa fatores de risco relacionados ao pequeno intervalo intergestacional. Sabe-se que, intervalos menores que dois anos prejudicam o binômio maternofetal e contribuem para gestações indesejadas (ANDRADE RD, et al., 2015).

Espaçar o nascimento entre os filhos pode reduzir a mortalidade materna em até $30 \%$ ao passo que, reduz a mortalidade infantil em $10 \%$. Além da redução da morbimortalidade, o espaçamento intergestacional auxilia a mulher na recuperação do organismo pré-gravídico, das condições biopsicossociais e evita que haja prejuízo na atenção aos cuidados com os primeiro filhos (DEV R, et al., 2019b).

O planejamento familiar foi instituído a partir da Lei № 9263 de 1996, a qual determina ações que regulam a fecundidade, orienta quanto a constituição da prole pelo casal e fornece assistencialismo quanto as questões reprodutivas e sexuais (BRASIL, 1996). Com isto, é de extrema relevância que informação de qualidade seja garantida não somente para a mulher, mas para o casal. $O$ aconselhamento sobre anticoncepção durante o período puerperal é de total relevância para que a mulher exerça com autonomia o seu direito de escolha. A qualidade da assistência prestada influencia na escolha e na continuidade do método a ser utilizado gerando melhores resultados (ABDULRESHID M e DADI HB, 2020).

Ter acesso não somente à informação, mas também à disponibilidade dos métodos contraceptivos torna o processo muito mais eficaz. Isso permite que haja um poder de escolha do método que mais se adequa às condições de vida daquela puérpera. A partir do momento em que profissionais capacitados estão disponíveis para fornecer tal conhecimento sobre anticoncepção, as mulheres se sentem mais seguras e dúvidas são sanadas, afim de que o retorno às atividades sexuais não seja prejudicado (DULLI LS, et al., 2016).

Deve-se considerar que cada mulher está inserida num contexto social diferente e que as individualidades devem ser priorizadas. Dessa forma, existem falhas no que se refere ao sistema de saúde, uma vez que, em diversos locais, uma variedade pequena de métodos ou a dificuldade de acesso a consultas com médicos ginecologistas é uma constante. O conhecimento sobre as características do método, quais suas contraindicações, vantagens e desvantagens são importantes para a garantia do direito reprodutivo da mulher e do casal (ANDRADE RD, et al., 2015).

REAC/EJSC | Vol. 14 | e4274 | DOI: https://doi.org/10.25248/reac.e4274.2020 Página 2 de 10 
Algumas barreiras são criadas ao uso da anticoncepção no pós parto devido a conceitos errôneos e medo de efeitos colaterais que essas medicações e dispositivos podem trazer. A desinformação propaga certos "mitos", o que dificulta ou cria resistência ao uso de determinado anticoncepcional. Portanto, prestar informação de qualidade desmistifica o uso da contracepção no pós parto e permite que as mulheres possam ter um retorno da atividade sexual sem o medo de uma nova gestação (DEV R, et al., 2019a).

O retorno à fecundidade muitas vezes não é compreendido entre as mulheres no pós parto, já que pode acontecer mesmo em amenorreia e, dessa forma, muitas delas podem conceber em um curto intervalo de tempo. Desse modo, a informação deve ser transmitida desde o período pré-natal e reforçada após o parto, para que essas puérperas possam estar seguras e atentas ao risco de uma nova gestação (BAQUI AH, et al., 2018).

A estratégia para melhorar o planejamento familiar no pós-parto é fazer com que o aconselhamento esteja inserido em todo o conjunto da atenção básica. Dessa forma, as mulheres seriam orientadas desde antes da concepção, passando pelo ciclo gravídico e pré-natal, o que reforçaria todas as medidas de anticoncepção tomadas durante o puerpério (CLELAND J, et al., 2015).

Nesse sentido, a pesquisa em questão justifica-se por evidenciar a importância do planejamento familiar no contexto do puerpério, levando em consideração que este é um momento oportuno para o aconselhamento contraceptivo e ressaltar como a baixa adesão às práticas de planejamento familiar tornam-se prejudiciais ao binômio maternofetal, ocasionando intervalos intergestacionais pouco espaçados e gestações não planejadas.

Com base nesse cenário, a pesquisa buscou identificar qual o impacto que o planejamento familiar e a anticoncepção trazem para a vida de mulheres no pós-parto e o quanto isso reflete na qualidade de vida.

\section{MÉTODOS}

Trata-se de uma revisão integrativa acerca do impacto que o planejamento familiar e a anticoncepção no pós parto desempenham na qualidade de vida de puérperas e casais. O levantamento sistematizado ocorreu entre os meses de maio e junho de 2020. O presente estudo baseou-se nos princípios metodológicos para busca de artigos nas bases de dados eletrônicas: Sistema Online de Busca e Análise de Literatura Médica (MEDLINE) e US National Library of Medicine/National Institutes of Health (PubMed).

Para o rastreamento dos artigos indexados nas bases de dados mencionadas acima foram utilizados os descritores cadastrados em Ciências da Saúde (DeCS) da BVS (Biblioteca Virtual de Saúde) que compõem o tema: "planejamento familiar", "anticoncepção" e "puerpério", sendo estes pesquisados de forma associada, utilizando o operador booleano AND.

Para eleger os artigos de interesse foi realizada uma leitura prévia do título e resumo, onde foram selecionados os textos com informações proveitosas para o trabalho. Em seguida, a busca consistiu em selecionar as obras de interesse de acordo com os critérios de inclusão e exclusão definidos anteriormente a partir da leitura seletiva dos resumos. E, por fim, a etapa de extração de dados, que permitiu analisar minuciosamente os artigos na íntegra, selecionando aquelas publicações que tratavam de assuntos relevantes ao tema.

Os critérios de inclusão definidos para seleção dos artigos foram: artigos publicados em português, inglês e espanhol; que abordassem a importância do planejamento familiar e anticoncepção no puerpério, mesmo que este não seja o tema principal do trabalho; artigos completos e disponíveis, sendo citados no mínimo dois descritores utilizados no estudo e aqueles publicados nos últimos cinco anos (2015-2019).

Foram excluídos do trabalho a falta de adequação do objetivo do estudo; artigos não indexados nas bases de dados; artigos duplicados, bem como aqueles que disponibilizaram somente os resumos da publicação.

Após realização da busca, os artigos foram exportados e agrupados no gerenciador de referências Zotero para análise e exclusão de duplicatas de forma automatizada.

REAC/EJSC | Vol. 14 | e4274 | DOI: https://doi.org/10.25248/reac.e4274.2020 Página 3 de 10 


\section{RESULTADOS}

$\mathrm{Na}$ base de dados PubMed, combinaram-se os descritores da seguinte forma: planejamento familiar AND anticoncepção AND puerpério, obtendo-se 242 artigos. Foram selecionados 50 artigos a partir da leitura de títulos e resumos. Sendo feita a leitura na íntegra e considerando critérios de inclusão e exclusão, 13 artigos foram selecionados para estudo.

Já na base de dados Medline, a combinação dos descritores citados acima resultou em 212 artigos. Quando adicionado o ano de publicação, foram encontrados 16 artigos. Excluindo-se artigos duplicados e aqueles que, ainda assim, não se encaixavam no tema de acordo com os critérios de inclusão e exclusão, 3 artigos foram selecionados para estudo.

Como havia artigos repetidos entre as bases de dados PubMed e Medline, foram mantidos os que estavam indexados na base de dados PubMed. Com isso, foram selecionados no total 16 artigos para leitura de texto completo, sendo todos eles incluídos para integrar esta revisão sistemática. A Figura 1 ilustra fluxograma que descreve as etapas de seleção dos estudos.

Figura 1 - Fluxograma com as etapas de seleção dos artigos.
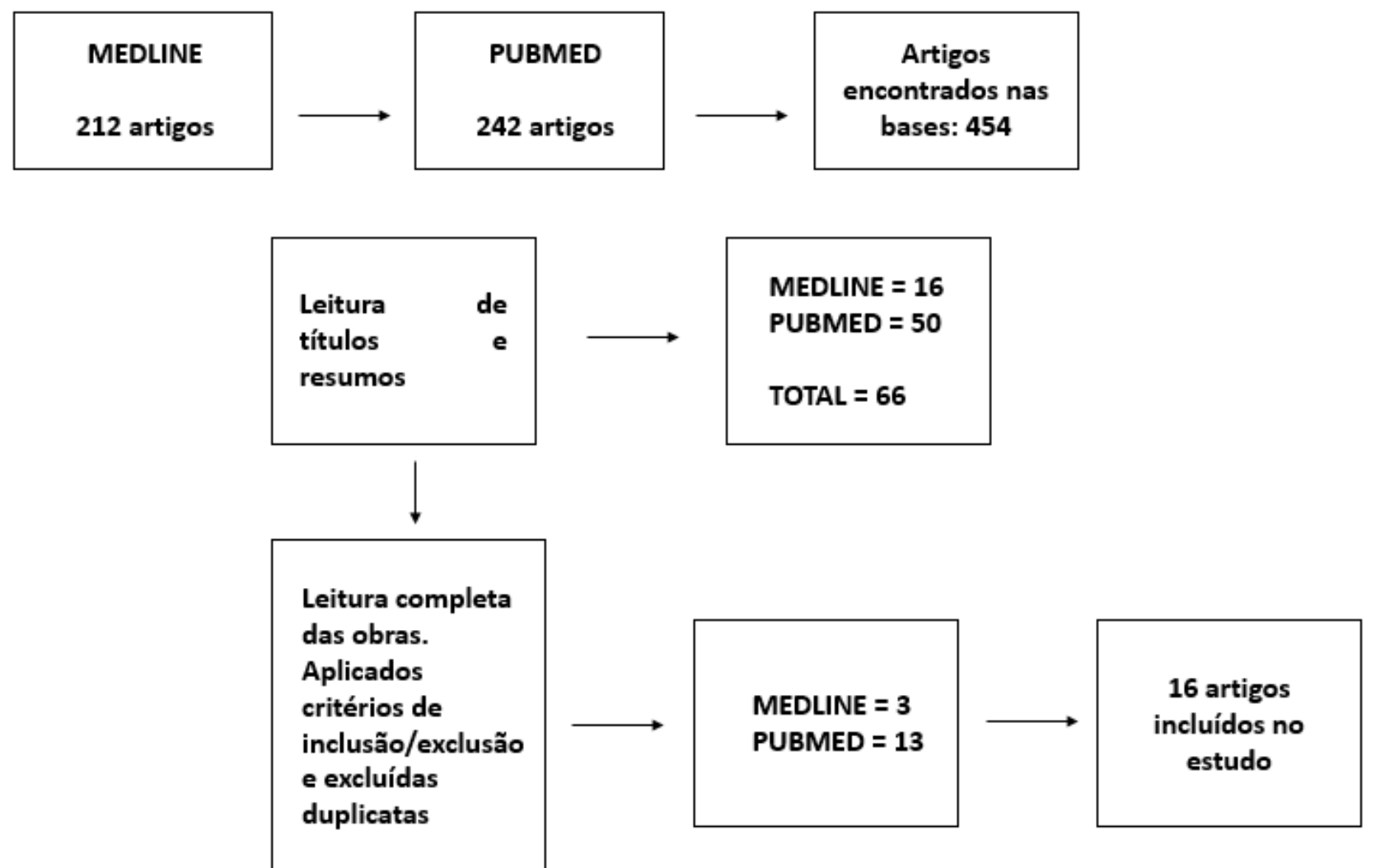

Fonte: Eloy CVB, et al., 2020.

Após seleção dos estudos, eles foram organizados de acordo com os autores, ano de publicação, objetivos e resultados. Todos os artigos foram publicados em inglês. Com relação ao ano de publicação, 2015 apresenta sete $(43,75 \%)$ artigos publicados; 2016 com três $(18,75)$ artigos; 2017 com um artigo $(6,25 \%)$; 2018 , três artigos (18,75\%) e 2019, dois artigos (12,5\%). A Quadro 1 mostra os 16 artigos selecionados e organizados conforme explicitado anteriormente. 
Quadro 1 - Artigos recuperados na revisão sistemática.

\begin{tabular}{|c|c|c|c|}
\hline Autor & Ano de publicação & Objetivos & Resultados \\
\hline Dev R, et al. & 2019 & $\begin{array}{c}\text { Identificar as necessidades não atendidas de } \\
\text { planejamento familiar pós parto e quais são as } \\
\text { barreiras e facilitadores da anticoncepção pós natal, } \\
\text { de modo a criar estratégias que permitam espaçar } \\
\text { as gestações. }\end{array}$ & $\begin{array}{c}\text { O uso de planejamento familiar foi baixo durante o } \\
\text { primeiro ano pós parto, ao passo que, foi alto o desejo } \\
\text { de evitar novas gestações em intervalos menores que } \\
\text { dois anos. }\end{array}$ \\
\hline Dev R, et al. & 2019 & $\begin{array}{c}\text { Entrevistar puérperas e prestadores de } \\
\text { planejamento familiar para avaliar a aceitabilidade e } \\
\text { viabilidade de um aplicativo interativo para escolha } \\
\text { da contracepção pós parto. }\end{array}$ & $\begin{array}{l}\text { O aplicativo auxilia na decisão do contraceptivo baseado } \\
\text { em um algoritmo que fornece aconselhamento sobre } \\
\text { anticoncepção. }\end{array}$ \\
\hline Tafere TE, et al. & 2018 & $\begin{array}{l}\text { Trazer informações sobre o papel do } \\
\text { aconselhamento acerca do planejamento familiar } \\
\text { ainda durante as visitas de pré-natal e o quanto isso } \\
\text { impacta na aderência ao uso de métodos } \\
\text { contraceptivos no pós parto. }\end{array}$ & $\begin{array}{l}\text { A satisfação nos serviços de atendimento pré-natal, } \\
\text { aconselhamento sobre preparação e prevenção a } \\
\text { complicações no pós parto, além de aconselhamento ao } \\
\text { aleitamento materno foram preditores independentes ao } \\
\text { uso de contracepção pós parto obtendo efeito } \\
\text { significativo na adesão ao planejamento familiar. }\end{array}$ \\
\hline Gebremedhin AY, et al. & 2018 & $\begin{array}{c}\text { O uso de planejamento familiar pós parto e seus } \\
\text { fatores associados entre mulheres em período pós } \\
\text { parto prolongado. }\end{array}$ & $\begin{array}{l}\text { O estudo constatou que a prevalência de planejamento } \\
\text { familiar pós parto foi alta e que o estado civil, número de } \\
\text { meses pós parto, histórico de retomada da menstruação } \\
\text { após o nascimento e histórico de uso de contraceptivos } \\
\text { antes da gravidez atual foram fatores significativamente } \\
\text { associados ao uso de contracepção pós parto. }\end{array}$ \\
\hline Baqui $\mathrm{AH}$, et al. & 2018 & $\begin{array}{c}\text { Examinar a viabilidade de integrar um pacote de } \\
\text { intervenção de planejamento familiar pós-parto em } \\
\text { um pacote de intervenção de saúde materna e } \\
\text { neonatal. }\end{array}$ & $\begin{array}{l}\text { A integração do planejamento familiar pós parto no } \\
\text { pacote de intervenção não influenciou negativamente os } \\
\text { resultados maternos e neonatais }\end{array}$ \\
\hline $\begin{array}{l}\text { Sonalkar S e Gaffield } \\
\text { ME }\end{array}$ & 2017 & $\begin{array}{l}\text { Criar uma plataforma para orientação da OMS sobre } \\
\text { planejamento familiar pós parto para uso de clínicos, } \\
\text { gerente de programas e formuladores de políticas. }\end{array}$ & $\begin{array}{l}\text { Limitação do idioma inglês, conexão com internet, além } \\
\text { de outras barreiras ao uso da contracepção pós parto } \\
\text { como custo, acesso, confidencialidade e tolerância aos } \\
\text { efeitos adversos também precisam ser abordados nessa } \\
\text { ferramenta. }\end{array}$ \\
\hline
\end{tabular}

REAC/EJSC | Vol. 14 | e4274 | DOI: https://doi.org/10.25248/reac.e4274.2020 Página 5 de 10 


\begin{tabular}{|c|c|c|c|}
\hline Achyut $P$, et al. & 2016 & $\begin{array}{c}\text { Analisar até que ponto as informações sobre } \\
\text { planejamento familiar são integradas aos serviços } \\
\text { de saúde materna e quais os efeitos da prestação } \\
\text { de informações no uso de contraceptivos modernos } \\
\text { durante o pós parto. }\end{array}$ & $\begin{array}{c}\text { O estudo mostrou que informações prestadas ainda no } \\
\text { pré-natal, durante o } 3^{0} \text { trimestre, tem maior impacto no } \\
\text { uso de contracepção pós parto. }\end{array}$ \\
\hline Dulli LS, et al. & 2016 & $\begin{array}{l}\text { Testar a eficácia do aconselhamento em } \\
\text { planejamento familiar nos serviços de imunização } \\
\text { infantil, a fim de aumentar o uso de métodos } \\
\text { contraceptivos entre as mulheres no pós parto. }\end{array}$ & $\begin{array}{c}\text { Analisados dados de } 825 \text { mulheres do grupo intervenção } \\
\text { e } 829 \text { do grupo controle, notou-se que ambos os grupos } \\
\text { apoiaram predominantemente o conceito de integração } \\
\text { dos componentes de planejamento familiar nos serviços } \\
\text { de imunização ( } 97,9 \% \text { em cada grupo). }\end{array}$ \\
\hline Blazer C e Prata N & 2016 & $\begin{array}{c}\text { Avaliar as estratégias de intervenção recentes que } \\
\text { apresentam uma maior promessa de diminuir as } \\
\text { necessidades não atendidas de contracepção em } \\
\text { mulheres pós parto. }\end{array}$ & $\begin{array}{l}\text { O envolvimento de parceiros masculinos, a integração } \\
\text { com outras formas de prestação de serviço, como a } \\
\text { imunização e programas inovadores de entrega de } \\
\text { produtos podem aumentar o conhecimento e diminuir as } \\
\text { taxas de gravidez. }\end{array}$ \\
\hline Sileo KM, et al. & 2015 & $\begin{array}{l}\text { Explorar os fatores determinantes para adoção do } \\
\text { planejamento familiar e uso de contracepção entre } \\
\text { mulheres pós parto na zona rural de Uganda. }\end{array}$ & $\begin{array}{c}\text { Fatores como educação, uso prévio de contraceptivos, } \\
\text { comunicação com o parceiro e necessidade percebida } \\
\text { de planejamento familiar foram identificados como } \\
\text { determinantes e devem ser considerados no } \\
\text { planejamento pós parto. }\end{array}$ \\
\hline Cleland J, et al. & 2015 & $\begin{array}{c}\text { O artigo é uma revisão de literatura que buscou } \\
\text { discutir a importância das intervenções sobre o uso } \\
\text { de métodos contraceptivos após o parto. }\end{array}$ & $\begin{array}{l}\text { Necessidade de adoção imediata de planejamento } \\
\text { familiar pós parto, incentivo a amenorreia lactacional por } \\
\text { um período de seis meses, iniciar contracepção até seis } \\
\text { semanas após o parto constituem estratégias eficazes } \\
\text { para aumentar o intervalo entre a gestações. }\end{array}$ \\
\hline Moore Z, et al. & 2015 & $\begin{array}{c}\text { Analisar dados de pesquisas demográficas recentes } \\
\text { para examinar padrões de intervalos entre } \\
\text { gestações, risco de gravidez e uso de métodos de } \\
\text { planejamento familiar entre mulheres com } 0-23 \\
\text { meses pós parto. }\end{array}$ & $\begin{array}{l}50 \% \text { ou mais dos nascimentos não primários ocorrem } \\
\text { em intervalos curtos. A necessidade geral não atendida } \\
\text { de planejamento familiar pelas puérperas não mudou de } \\
\text { forma considerável desde a análise feita em } 2001 \text { e } \\
\text { continua universalmente alto. }\end{array}$ \\
\hline
\end{tabular}

$\overline{\text { REAC/EJSC | Vol. } 14 \text { | e4274 | DOI: https://doi.org/10.25248/reac.e4274.2020 Página } 6 \text { de } 10}$ 


\begin{tabular}{|c|c|c|c|}
\hline Hounton S, et al. & 2015 & $\begin{array}{l}\text { Avaliar padrões e tendências no uso do } \\
\text { planejamento familiar pós parto (PFPP), determinar } \\
\text { se o PFPP está associado ao intervalo } \\
\text { intergestacional e identificar os serviços de saúde } \\
\text { mais intimamente associados ao PFPP. }\end{array}$ & $\begin{array}{l}\text { Os resultados indicam que a melhoria do planejamento } \\
\text { familiar pós-parto requer políticas e estratégias para lidar } \\
\text { com as desigualdades causadas por fatores } \\
\text { socioeconômicos e a integração do planejamento } \\
\text { familiar com os serviços de saúde materna e neonatal. }\end{array}$ \\
\hline Ayiasi RM, et al. & 2015 & $\begin{array}{c}\text { Avaliar o efeito do aconselhamento contraceptivo } \\
\text { pré natal no uso de contracepção pós parto e nos } \\
\text { resultados da gravidez após um ano. }\end{array}$ & $\begin{array}{c}\text { Cerca de metade das mães em cada braço considerou } \\
\text { adiar a próxima gravidez } 47,1 \% \text { (controle) e } 49 \% \\
\text { (intervenção). Desses } 71,4 \% \text { no controle e } 87 \% \text { na } \\
\text { intervenção consideraram usar um método contraceptivo } \\
\text { moderno, apenas } 28,2 \% \text { do controle e } 31,6 \% \text { na } \\
\text { intervenção eram atuais usuários contraceptivos } \\
\text { modernos. }\end{array}$ \\
\hline Pasha $O$, et al. & 2015 & $\begin{array}{c}\text { Avaliar a intenção de fertilidade, o uso de } \\
\text { contraceptivos e a necessidade não atendida de } \\
\text { planejamento familiar entre as mulheres, bem como } \\
\text { identificar as que apresentam maior risco de ter uma } \\
\text { necessidade não atendida de planejamento familiar } \\
\text { durante esse período. }\end{array}$ & $\begin{array}{c}\text { Menos de } 5 \% \text { dessas mulheres desejavam ter outra } \\
\text { gravidez dentro do ano. Apesar disso, as necessidades } \\
\text { não atendidas variaram de } 25 \% \text { a } 96 \% \text {. Mesmo entre os } \\
\text { usuários de contraceptivos modernos, a captação dos } \\
\text { contraceptivos reversíveis de ação mais eficaz de longa } \\
\text { duração foi baixa. }\end{array}$ \\
\hline Rossier C, et al. & 2015 & $\begin{array}{l}\text { Avaliar indicadores de aconselhamento pós parto } \\
\text { em mulheres expostas ao risco de novas gestações }\end{array}$ & $\begin{array}{l}\text { Embora a proteção oferecida pela abstinência pós-parto } \\
\text { e amenorreia lactacional diminua a necessidade não } \\
\text { atendida, análises posteriores mostram que as mulheres } \\
\text { também costumam confiar nesses métodos sem serem } \\
\text { realmente protegidas. }\end{array}$ \\
\hline
\end{tabular}

Fonte: Eloy CVB, et al., 2020. 


\section{DISCUSSÃO}

O planejamento familiar (PF) no puerpério é uma garantia dos direitos reprodutivos das mulheres e do casal e garante autonomia quanto ao poder de escolha acerca da contracepção pós parto. No entanto, a maioria das mulheres, principalmente em países de baixa e média renda, não são atendidas quanto ao aconselhamento contraceptivo pós parto e muitas delas relatam desejo de impedir novas gestações por um período de pelo menos um ano. Sabe-se que intervalos entre gestações menores que 24 meses apresentam alta morbimortalidade materno infantil e o risco de gravidez indesejada é alto durante esse período (DEV R, et al., 2019b).

A não utilização de métodos contraceptivos e a falta de acesso a informações e serviços de saúde que forneçam aconselhamento anticoncepcional de qualidade contribuem para a necessidade não atendida acerca do PF pós parto (DEV R, et al., 2019a). Segundo Sileo, et al. (2015) 37\% das mulheres têm uma necessidade não atendida de PF e apenas $27 \%$ relatam atualmente usar métodos contraceptivos eficazes. Esses dados estão em concordância com o que diz a Organização Mundial da Saúde, que aponta necessidades não atendidas como um problema de saúde pública já que, 20\% das mortes obstétricas são atribuídas ao não uso da contracepção moderna (SONALKAR S e GAFFIELD ME, 2017).

Existem alguns fatores que aumentam a necessidade do aconselhamento contraceptivo pós parto. Entre eles, a escolaridade constitui um forte determinante porque mulheres com menor grau de instrução estão mais propensas ao risco de pequenos intervalos intergestacionais (SILEO KM, et al., 2015). Além disso, mulheres solteiras tiveram $91 \%$ menos probabilidade de usar métodos contraceptivos quando comparadas a mulheres casadas. O estado civil tem uma influência importante pois, se a mulher é casada, a tendência é que ela possa ter contato sexual mais precoce pós parto, o que aumenta o risco de uma nova gestação. Outro achado determinante foi o fato de que, algumas mulheres não percebem a necessidade de iniciar $\mathrm{PF}$ e isso se deve à um provável reflexo das atitudes que muitos parceiros tem em relação à anticoncepção. Fica claro que 0 apoio masculino para boas práticas de contracepção no pós parto são fundamentais para 0 planejamento em saúde reprodutiva (GEBREMEDHIN AY, et al., 2018)

Entretanto, mulheres que já faziam o uso de métodos contraceptivos antes da última gestação tiveram maior probabilidade de continuar fazendo o uso no pós parto. Isso pode ser explicado pelo fato de elas terem um maior conhecimento do método o que melhora a atitude em relação ao uso no puerpério (GEBREMEDHIN AY, et al., 2018).

A não utilização de métodos contraceptivos no pós parto pode estar associada a equívocos sobre o risco de gravidez. Muitas mulheres relacionam o retorno da menstruação com 0 uso de contraceptivos, não sabendo que, podem obter o retorno da fertilidade mesmo em amenorreia (ACHYUT P, et al., 2016). Em um estudo em Bangladesh, 33\% das mulheres retomaram a atividade sexual dentro de 3 meses após o parto, enquanto a maioria era propensa a utilizar anticoncepção após o retorno da menstruação (BLAZER C e PRATA N, 2016).

Embora poucas puérperas relatem o uso da amenorreia lactacional (LAM) como método contraceptivo, muitas delas atendem aos critérios para a correta eficácia do método, estando, em grande parte, protegidas de uma gravidez por seis meses (ROSSIER C, et al., 2015). No entanto, qualquer alteração que houver no status da amamentação pode desencadear a necessidade de iniciar um outro método anticoncepcional (SONALKAR S e GAFFIELD ME, 2017). Mesmo aquelas que estão amamentando, $37 \%$ das mulheres sexualmente ativas têm risco aumentado durante os primeiros seis meses após o parto, aumentando para 64\% entre as mulheres de 6 a 11 meses pós parto (TAFERE TE, et al., 2018).

Hoje, uma das principais barreiras ao uso da contracepção pós parto é o conhecimento insuficiente acerca dos métodos contraceptivos. Isso acaba gerando uma série de mitos e conceitos errôneos, além do medo de possíveis efeitos colaterais. Informações sobre métodos que podem causar câncer ou infertilidade são bem difundidos e dificultam o uso principalmente de métodos de longa duração (AYIASI RM, et al., 2015).

Por isso, é importante que intervenções educacionais na prática de PF sejam adotadas a fim de dissipar conceitos que dificultam a utilização da anticoncepção pós parto (CLELAND J, et al., 2015). Uma estratégia

REAC/EJSC | Vol. 14 | e4274 | DOI: https://doi.org/10.25248/reac.e4274.2020 Página 8 de 10 
para melhorar o aconselhamento pós parto é incorporar o PF em todo o continuum da atenção em saúde reprodutiva. Isso quer dizer que, desde o pré-natal as mulheres devem ser orientadas quanto à prática da anticoncepção no puerpério (DULLI LS, et al., 2016).

A frequente exposição das gestantes a profissionais da saúde durante o pré-natal faz com que haja uma melhor oportunidade para discutir sobre PF, quando então, casais não estão distraídos pela chegada do novo bebê e têm, assim, mais tempo para considerar todas as opções (TAFERE TE, et al., 2018). Para Achyut $P$, et al. (2016) as mulheres que receberam informações sobre PF durante o terceiro trimestre de gestação tiveram 1,85 vezes mais chance de usar método contraceptivo moderno no pós parto.

Ainda permanece alta a necessidade não atendida de PF no pós parto. A lógica e o objetivo do aconselhamento familiar é fazer com que, a partir da informação de qualidade prestada, as mulheres possam escolher o método de acordo com as indicações e características que mais lhe agradam e fazer o uso por 2 anos ou mais, dependendo das intenções reprodutivas da mulher e do casal (HOUNTON S, et al., 2015). Mesmo quando há o uso de métodos contraceptivos no puerpério, a maioria se apoia predominantemente em métodos de curta ação. Ao implementar práticas de aconselhamento no pós parto imediato, a adoção de métodos de ação prolongada aumentou, desmistificando preconceitos acerca de determinados anticoncepcionais (MOORE Z, et al., 2015).

Sabe-se que intervalos intergestacionais maiores que 2 anos trazem vantagens para o binômio maternofetal. Crianças nascidas entre 3 a 4 anos após um nascimento anterior apresentam vantagem significativa de sobrevivência em comparação a crianças nascidas em menor intervalo de tempo (PASHA O, et al., 2015). Já intervalos curtos de nascimento costumam estar associados a baixo peso ao nascer, risco de aborto espontâneo, parto prematuro, anemia, ruptura de membranas entre outros eventos (BAQUI AH, et al., 2018).

\section{CONSIDERAÇÕES FINAIS}

O planejamento familiar (PF) pós parto constitui um importante instrumento nas práticas de saúde reprodutiva das mulheres. Apesar do déficit que existe quanto ao aconselhamento pós parto, o PF, quando bem orientado, fornece assistência de qualidade permitindo que, a mulher ou o casal, tenham autonomia para decidir sobre as próximas gestações. Os trabalhos aqui incluídos demonstraram que as necessidades não atendidas de PF ainda são altas, o que pode favorecer desfechos adversos ao binômio maternofetal considerando pequenos intervalos intergestacionais. Dessa forma, torna-se essencial ampliar as estratégias de aconselhamento puerperal de modo a esclarecer sobre potenciais dúvidas acerca dos métodos contraceptivos, desmistificando preconceitos sobre anticoncepção pós parto, reforçando o uso da amenorreia lactacional e expondo os riscos de novas gestações. A partir desse estudo, fica claro que tratar questões acerca do PF vai muito além de somente orientar qual melhor método anticoncepcional, mas entender individualmente cada mulher e compreender o quanto isso impacta na saúde pública e na qualidade de vida.

\section{REFERÊNCIAS}

1. ABDULRESHID M, DADI HB. Assessment of Family Planning Counseling Provided for Postpartum Women and Associated Factors. International Journal of Reproductive Medicine, 2020.

2. ACHYUT P, et al. Integration of family planning with maternal health services: an opportunity to increase postpartum modern contraceptive use in urban Uttar Pradesh, India. The Journal of Family Planning and Reproductive Health Care, 2016; v. 42, n. 2, p. 107-115.

3. ANDRADE RD, et al. Fatores relacionados à saúde da mulher no puerpério e repercussões na saúde da criança. Escola Anna Nery, 2015; v. 19, n. 1, p. 181-186.

4. AYIASI RM, et al. The effect of prenatal counselling on postpartum family planning use among early postpartum women in Masindi and Kiryandongo districts, Uganda. The Pan African Medical Journal, 2015; v. 21, p. 138.

5. BAQUI AH, et al. Impact of integrating a postpartum family planning program into a community-based maternal and newborn health program on birth spacing and preterm birth in rural Bangladesh. Journal of Global Health, 2018; v. 8, n. 2, p. 020406.

REAC/EJSC | Vol. 14 | e4274 | DOI: https://doi.org/10.25248/reac.e4274.2020 Página 9 de 10 
6. BLAZER C, PRATA N. Postpartum family planning: current evidence on successful interventions. Open Access Journal of Contraception, 2016; v. 7, p. 53-67, 11.

7. BRASIL. Lei no 9.263. Presidência da República, 12 de janeiro de 1996. Disponível em: <http://www.planalto.gov.br/ccivil_03/leis/l9263.htm>. Acesso em: 30 de maio 2020.

8. CLELAND J, et al. Interventions to Improve Postpartum Family Planning in Low- and Middle-Income Countries: Program Implications and Research Priorities. Studies in Family Planning, 2015; v. 46, n. 4, p. 423-441.

9. DEV R, et al. Acceptability, feasibility and utility of a Mobile health family planning decision aid for postpartum women in Kenya. Reproductive Health, 2019a; v. 16.

10. DEV R, et al. A systematic review and meta-analysis of postpartum contraceptive use among women in low- and middle-income countries. Reproductive Health, 2019b; v. 16.

11. DULLI LS, et al. Meeting Postpartum Women's Family Planning Needs Through Integrated Family Planning and Immunization Services: Results of a Cluster-Randomized Controlled Trial in Rwanda. Global Health: Science and Practice, 2016; v. 4, n. 1, p. 73-86.

12. GEBREMEDHIN AY, et al. Family planning use and its associated factors among women in the extended postpartum period in Addis Ababa, Ethiopia. Contraception and Reproductive Medicine, 2018; v. 3.

13. HOUNTON S, et al. Patterns and trends of postpartum family planning in Ethiopia, Malawi, and Nigeria: evidence of missed opportunities for integration. Global Health Action, 2015; v. 8.

14. MOORE Z, et al. Missed opportunities for family planning: an analysis of pregnancy risk and contraceptive method use among postpartum women in 21 low- and middle-income countries. Contraception, 2015; v. 92, n. 1, p. 31-39.

15. PASHA O, et al. Postpartum contraceptive use and unmet need for family planning in five low-income countries. Reproductive Health, 2015; v. 12, n. Suppl 2, p. S11.

16. ROSSIER C, et al. Reassessing Unmet Need for Family Planning in the Postpartum Period. Studies in Family Planning, 2015; v. 46, n. 4, p. 355-367.

17. SILEO KM, et al. Determinants of family planning service uptake and use of contraceptives among postpartum women in rural Uganda. International journal of public health, 2015; v. 60, n. 8, p. 987-997.

18. SONALKAR S, GAFFIELD ME. Introducing the World Health Organization Postpartum Family Planning Compendium. International Journal of Gynaecology and Obstetrics, 2017; v. 136, n. 1, p. 2-5.

19. TAFERE TE, et al. Counseling on family planning during ANC service increases the likelihood of postpartum family planning use in Bahir Dar City Administration, Northwest Ethiopia: a prospective follow up study. Contraception and Reproductive Medicine, 2018; v. 3, 27.

20. ZUGAIB, M. Obstetrícia Zugaib. 3ae edição. São Paulo: Editora Manole, 2016; 450p. 